\title{
On arbitrage and Markovian short rates in fractional bond markets
}

\author{
Pavel V. Gapeev*
}

\begin{abstract}
We study a bond market model and related term structure of interest rates driven by a fractional Brownian motion with self-similarity parameter $H \in(1 / 2,1)$. We present a criterion on the deterministic forward rate volatility under which the short rate process is Markovian and construct an admissible self-financing portfolio realizing an arbitrage opportunity.
\end{abstract}

\section{Introduction}

One of the interesting problems in financial mathematics is the developing of stochastic theory of interest rates and related bond market models. The general methodology for studying term structures of interest rates proposed by Heath, Jarrow and Morton [13] was based on the investigation of a model with forward rates driven by a multi-dimensional Wiener process. This model was extended by Björk, Kabanov and Runggaldier [1], who supposed the driving process to be a sum of a diffusion and a marked point process having finitely many jumps on finite time intervals, introduced the notion of admissible self-financing portfolios and studied the questions of absence of arbitrage and existence of equivalent martingale measures on bond markets.

Another question of interest is to find conditions under which the short rate process is Markovian, since in that case the bond price gets a simpler representation. This question for the bond market model driven by a Wiener process was studied by Carverhill [3]. It was proved that the short rate process is Markovian within the Heath-Jarrow-Morton framework with a deterministic volatility function if and only if this volatility factorizes into a product of two functions only depending on the current time and maturity time, respectively. This result was generalized by Eberlein and Raible [8] and Küchler and Naumann [15] for models driven by Lévy processes, and by Gapeev and Küchler [11] for mixed jump-diffusion models.

In the recent years fractional Brownian motion was used for modelling assets on financial markets. Originally introduced and studied by Kolmogorov [14] this process was named by Mandelbrot and van Ness [19]. For a complete study of the properties of fractional Browinan

*Previous address: Russian Academy of Sciences, Institute of Control Sciences, 117997, Russia, Moscow, Profsoyuznaya Street 65; Current address: London School of Economics, Department of Mathematics, Houghton Street, London WC2A 2AE, United Kingdom, e-mail: p.v.gapeev@lse.ac.uk

Mathematics Subject Classification 2000. Primary 60G15, 60G18, 91B70. Secondary 60J25, 60H05, $91 \mathrm{~B} 28$.

Key words and phrases: Bond market model, term structure of interest rates, Heath-Jarrow-Morton approach, fractional Brownian motion, fundamental martingale, prediction formula, average risk neutral measure, pathwise stochastic integration, arbitrage opportunity. 
motion we refer to Decreusefond and Üstünel [6] and Samorodnitsky and Taqqu [25]. Being a non-semimartingale fractional Brownian motion implies the existence of arbitrage opportunities in the related financial market models. Rogers [23] proposed an arbitrage strategy based on the self-similarity of this process and the derived in [19] representation with respect to a Wiener process on an infinite time interval. Shiryaev [26; Chapter VII, Section 2c] (see also Dasgupta and Kallianpur [7]) presented an explicit (self-financing) portfolio realizing an arbitrage opportunity by means of the pathwise stochastic integration (cf. Föllmer [9], Föllmer, Protter and Shiryaev [10], Lin [17] and Zähle [31]). The question of the existence of arbitrage in models with fractional Brownian motion was also studied in Salopek [24], Norvaiša [22], Sottinen [27] and Cheridito [4]. Valkeila [30] presented some analytical results for geometric fractional Brownian motion and introduced the notion of average risk neutral measure for a fractional stock market. For a complete review of results on financial market models driven by fractional Brownian motions we refer to Sottinen and Valkeila [28]-[29].

In the present paper we consider a bond market model and a term structure of interest rates of Heath-Jarrow-Morton type driven by a fractional Brownian motion with self-similarity parameter $H \in(1 / 2,1)$. We give a necessary and sufficient condition on the deterministic forward rate volatility under which the short rate is a Markov process. The Markov property of the short rate implies the simplification of conditional expectation in the corresponding prediction formula for the bond price under an average risk neutral measure. Moreover, the Markovian short rate being the same time a Gaussian process often turns out to be a semimartingale. (For a criterion for a Gaussian process with stationary increments to be a semimartingale see Cheridito [5].) By constructing an explicit admissible self-financing arbitrage portfolio we show that a bond market model with the short rate being a semimartingale can admit an arbitrage opportunity.

The paper is organized as follows. In Section 2 we formulate a fractional bond market model with the corresponding term structure of interest rates. In Section 3 we derive a prediction formula for the bond price under an average risk neutral measure. In Section 4 we prove a criterion for the short rate to be Markovian in the case of deterministic forward rate volatility. In Section 5 we construct an admissible self-financing portfolio realizing arbitrage.

\section{The fractional bond market model}

Suppose that on some stochastic base $\left(\Omega, \mathcal{F},\left(\mathcal{F}_{t}\right)_{t \in\left[0, T^{*}\right]}, Q\right)$ with a fixed time horizon $T^{*}>$ 0 there exists a fractional Brownian motion $Z=\left(Z_{t}\right)_{t \in\left[0, T^{*}\right]}$ with self-similarity parameter (Hurst exponent) $H \in(1 / 2,1)$, i.e. it is a continuous Gaussian process with $E\left[Z_{t}\right]=0$ and $E\left[Z_{t} Z_{s}\right]=\left(|t|^{2 H}+|s|^{2 H}-|t-s|^{2 H}\right) / 2$ for all $t, s \geq 0$, and let $\left(\mathcal{F}_{t}\right)_{t \in\left[0, T^{*}\right]}$ be its natural filtration: $\mathcal{F}_{t}=\sigma\left\{Z_{s} \mid 0 \leq s \leq t\right\}, t \in\left[0, T^{*}\right]$. Note that a fractional Brownian motion with $H \in(1 / 2,1)$ is a Dirichlet process (i.e. a process with sample paths of zero quadratic variation) and fails to be a semimartingale or a Markov process (see e.g. [18; Chapter IV, Section 9]).

Let us consider a term structure of bond prices $\left\{P(t, T) \mid 0 \leq t \leq T \leq T^{*}\right\}$ where the (positive) process $P=(P(t, T))_{t \in[0, T]}$ satisfying the normalization condition:

$$
P(T, T)=1
$$

denotes the price of a zero coupon bond at time $t$ maturing at time $T$ for each $T \in\left[0, T^{*}\right]$. 
Assuming that for fixed $t \in[0, T]$ the bond price $P(t, T)$ is $(Q$-a.s.) continuously differentiable in the variable $T$ on $\left[0, T^{*}\right]$, let us introduce the corresponding term structure of interest rates $\left\{f(t, T) \mid 0 \leq t \leq T \leq T^{*}\right\}$ where:

$$
f(t, T)=-\frac{\partial \log P(t, T)}{\partial T}
$$

is the instantaneous forward rate contracted at time $t$ for maturity $T$. On the other hand, integrating equation (2.2) and using condition (2.1), we get:

$$
P(t, T)=\exp \left(-\int_{t}^{T} f(t, u) d u\right)
$$

for all $0 \leq t \leq T \leq T^{*}$, and hence, we see the one-to-one correspondence between the bond prices and the forward rates. Let us also define the short rate process $r=(r(t))_{t \in\left[0, T^{*}\right]}$ by:

$$
r(t)=f(t, t)
$$

being the forward rate at time $t$ for maturity $t$, and the associated with it money account process $B=(B(t))_{t \in\left[0, T^{*}\right]}$ by:

$$
B(t)=\exp \left(\int_{0}^{t} r(s) d s\right)
$$

playing the role of numéraire in the model.

We will assume that for fixed $T \in\left[0, T^{*}\right]$ the forward rate process $f=(f(t, T))_{t \in[0, T]}$ is given by the expression:

$$
f(t, T)=f(0, T)+\int_{0}^{t} \alpha(s, T) d s+\int_{0}^{t} \sigma(s, T) d Z_{s}
$$

so that the short rate process (2.4) takes the form:

$$
r(t)=f(0, t)+\int_{0}^{t} \alpha(s, t) d s+\int_{0}^{t} \sigma(s, t) d Z_{s}
$$

where $\alpha(t, T)$ and $\sigma(t, T)$ are deterministic continuous functions on the triangle $\{(t, T) \mid 0<$ $\left.t<T \leq T^{*}\right\}$ such that the condition:

$$
\int_{0}^{T}|\alpha(t, T)| d t+\int_{0}^{T} \int_{0}^{T}|\sigma(t, T) \sigma(s, T)||t-s|^{2 H-2} d s d t<\infty
$$

holds for all $0<T \leq T^{*}$, and hence, the integrals with respect to $d Z_{s}$ are well-defined in the sense of $L^{2}(\Omega, \mathcal{F}, P)$ (see [21; Section 2] and references therein). We will also suppose that we are allowed (by the regularity of the functions) to interchange the order of integration.

Integrating expression (2.6) and applying arguments from [1; Section 2], we easily get that the bond price (2.3) admits the representation:

$$
\begin{aligned}
P(t, T) & =\frac{P(0, T)}{P(0, t)} \exp \left(-\int_{t}^{T} \int_{0}^{t} \alpha(s, u) d s d u-\int_{t}^{T} \int_{0}^{t} \sigma(s, u) d Z_{s} d u\right) \\
& =P(0, T) \exp \left(\int_{0}^{t} r(s) d s-\int_{0}^{t} \int_{s}^{T} \alpha(s, u) d u d s-\int_{0}^{t} \int_{s}^{T} \sigma(s, u) d u d Z_{s}\right) .
\end{aligned}
$$




\section{The prediction formula}

In order to derive the corresponding prediction formula for the bond price we will use the fact that the process $Z=\left(Z_{t}\right)_{t \in\left[0, T^{*}\right]}$ admits the representations:

$$
\begin{aligned}
Z_{t} & =2 H(H-1 / 2) \int_{0}^{t} \int_{u}^{t} s^{H-1 / 2}(s-u)^{H-3 / 2} d s d M_{u} \\
& =2 H(H-1 / 2) \int_{0}^{t} s^{H-1 / 2} \int_{0}^{s}(s-u)^{H-3 / 2} d M_{u} d s
\end{aligned}
$$

(cf. [21; Theorem 5.2]), where the process $M=\left(M_{t}\right)_{t \in\left[0, T^{*}\right]}$ defined by:

$$
M_{t}=c_{2} \int_{0}^{t} s^{1 / 2-H}(t-s)^{1 / 2-H} d Z_{s}
$$

is a fundamental martingale, i.e. a continuous Gaussian martingale with independent increments such that $E\left[M_{t}\right]=0,\langle M\rangle_{t}=c_{2}^{2} t^{2-2 H}, c_{2}^{2}=\Gamma(3 / 2-H) /(2 H \Gamma(3-2 H) \Gamma(H+1 / 2))$, whose natural filtration coincides with $\left(\mathcal{F}_{t}\right)_{t \in\left[0, T^{*}\right]}$ (see [20] and [21]).

For simplicity, we will assume that $Q$ is an average risk neutral measure, or in other words, the discounted bond price process $(P(t, T) / B(t))_{t \in[0, T]}$ has constant expectation under $Q$ :

$$
E\left[\frac{P(t, T)}{B(t)}\right]=\frac{P(0, T)}{B(0)}
$$

for all $0 \leq t \leq T$ (for the definition of average risk neutral measure for a fractional model of stock market see e.g. [30] or [28]). By means of straightforward calculations it can be verified that the formula (3.3) holds if in (2.9) we take:

$$
\alpha(t, T)=\sigma(t, T) \int_{t}^{T} \sigma(t, u) d u \frac{t^{2 H-1}}{2 H}
$$

for all $0<t<T \leq T^{*}$ (compare with the formula (25) in [13] or with (24) in [1]). Hence, from (2.9) and (3.4) it follows that the bond price $P(t, T)$ takes the expression:

$$
P(t, T)=e^{K(t, T)} E\left[\exp \left(-\int_{t}^{T} r(s) d s\right) \mid \mathcal{F}_{t}\right]
$$

where we set:

$$
K(t, T)=\int_{t}^{T} \int_{s}^{T} \sigma(s, u) \int_{s}^{u} \sigma(s, v) d v d u \frac{s^{2 H-1}}{2 H} d s-\int_{0}^{t} \int_{s}^{T} \sigma(s, u) d u d Z_{s}+L(t, T)
$$

and

$$
L(t, T)=-\log E\left[\exp \left(-\int_{0}^{T} \int_{s}^{T} \sigma(s, u) d u d Z_{s}\right) \mid \mathcal{F}_{t}\right] .
$$

We will call expression (3.5) the prediction formula for the bond price process $(P(t, T))_{t \in[0, T]}$ under the average risk neutral measure $Q$ (for a prediction formula for fractional Brownian motion see [12] and for the corresponding prediction formula for stock price see [30] or [28]). 
To compute the conditional expectation in (3.7), using the representation (3.1), we get:

$$
\begin{aligned}
& \int_{0}^{t} \sigma(s, t) d Z_{s}=2 H(H-1 / 2) \int_{0}^{t} \sigma(s, t) s^{H-1 / 2} \int_{0}^{s}(s-u)^{H-3 / 2} d M_{u} d s \\
& =2 H(H-1 / 2) \int_{0}^{t} \int_{u}^{t} \sigma(s, t) s^{H-1 / 2}(s-u)^{H-3 / 2} d s d M_{u}=\int_{0}^{t} \gamma(u, t) d M_{u}
\end{aligned}
$$

where we set:

$$
\gamma(t, T)=2 H(H-1 / 2) \int_{t}^{T} \sigma(s, T) s^{H-1 / 2}(s-t)^{H-3 / 2} d s
$$

for all $0<t<T \leq T^{*}$, and by virtue of the assumption (2.8), we may conclude that:

$$
\int_{0}^{T}|\alpha(t, T)| d t+\int_{0}^{T} \gamma^{2}(t, T) d\langle M\rangle_{t}<\infty
$$

holds for all $0<T \leq T^{*}$. Then changing the order of integration, we obtain:

$$
\begin{aligned}
& \int_{0}^{T} \int_{s}^{T} \sigma(s, u) d u d Z_{s}=\int_{0}^{T} \int_{0}^{u} \sigma(s, u) d Z_{s} d u \\
& =\int_{0}^{T} \int_{0}^{u} \gamma(s, u) d M_{s} d u=\int_{0}^{T} \int_{s}^{T} \gamma(s, u) d u d M_{s}
\end{aligned}
$$

and hence, the function $L(t, T)$ takes the form:

$$
L(t, T)=\int_{0}^{t} \int_{s}^{T} \gamma(s, u) d u d M_{s}-\frac{1}{2} \int_{t}^{T}\left(\int_{s}^{T} \gamma(s, u) d u\right)^{2} d\langle M\rangle_{s} .
$$

Finally, changing the order of integration, we get:

$$
\begin{aligned}
& \int_{t}^{T}(s-t)^{1 / 2-H} \int_{s}^{T} \sigma(u, T) u^{H-1 / 2}(u-s)^{H-3 / 2} d u d s \\
& =\int_{t}^{T} u^{H-1 / 2} \sigma(u, T) \int_{t}^{u}(u-s)^{H-3 / 2}(s-t)^{1 / 2-H} d s d u
\end{aligned}
$$

and therefore, using the fact that the last inner integral in (3.13) equals $\Gamma(H-1 / 2) \Gamma(3 / 2-H)$ (cf. [21; Lemma 2.2]), we obtain:

$$
L(t, T)=\int_{0}^{t} \delta(s, t ; T) d Z_{s}-\frac{1}{2} \int_{t}^{T}\left(\int_{s}^{T} \gamma(s, u) d u\right)^{2} d\langle M\rangle_{s}
$$

where we set:

$$
\delta(s, t ; T)=-\frac{s^{1 / 2-H}}{2 H \Gamma(H+1 / 2) \Gamma(3 / 2-H)} \frac{d}{d s} \int_{s}^{t}(v-s)^{1 / 2-H} \int_{v}^{T} \gamma(v, u) d u d v
$$

for all $0<s<t \leq T$.

Remark 3.1. We observe that from the arguments above it follows that in the case when the condition (3.4) is satisfied the bond price process $(P(t, T))_{t \in[0, T]}$ can be equivalently defined by the expression (3.5). We also note that if the short rate $(r(t))_{t \in\left[0, T^{*}\right]}$ turns out to be an $\left(\mathcal{F}_{t}, Q\right)$ - Markov process, then from (3.5) we get that the bond price admits the following representation:

$$
P(t, T)=e^{K(t, T)} E\left[\exp \left(-\int_{t}^{T} r(s) d s\right) \mid r(t)\right] .
$$




\section{Markovian short rates}

In order to study the question of the short rate being Markovian, let us first define the process $X=(X(t))_{t \in\left[0, T^{*}\right]}$ by:

$$
X(t)=\int_{0}^{t} \sigma(s, t) d Z_{s}
$$

which, by virtue of the arguments in (3.8), takes the form:

$$
X(t)=\int_{0}^{t} \gamma(s, t) d M_{s}
$$

with $\gamma(t, T)$ given by (3.9) and satisfying (3.10) for all $0<T \leq T^{*}$.

We continue with formulating two assertions being extensions of the corresponding results in [3], [8] and [15] (see also [11]).

Lemma 4.1. Let $(X(t))_{t \in\left[0, T^{*}\right]}$ be an $\left(\mathcal{F}_{t}, Q\right)$ - Markov process. Then for all $0<T \leq S \leq$ $T^{*}$ there is a measurable function $G$ such that:

$$
\int_{0}^{T} \gamma(t, S) d M_{t}=G\left(\int_{0}^{T} \gamma(t, T) d M_{t}\right) \quad(Q-\text { a.s. }) .
$$

Lemma 4.2. Let $\left(M_{t}\right)_{t \in[0, T]}$ be a fundamental martingale. Suppose that $f(t)$ and $g(t)$ are continuous functions on $(0, T)$, and for a measurable function $G$ we have:

$$
\int_{0}^{T} f(t) d M_{t}=G\left(\int_{0}^{T} g(t) d M_{t}\right) \quad(Q-\text { a.s. }) .
$$

Then there is a constant $c \in \mathbb{R}$ such that $f(t)=c \cdot g(t)$ for all $0<t<T$.

Since the process $\left(M_{t}\right)_{t \in\left[0, T^{*}\right]}$ is a Gaussian martingale with independent increments and the assumption (3.10) is satisfied for all $0<T \leq T^{*}$, the proofs of these two assertions can be done by means of the same arguments as in [8; Lemma 4.2] and [15; Lemma 3.1], respectively.

Now we formulate and prove the criterion for the short rate to be Markovian. Actually, we extend the results of [3], [8], [15] and [11] to the case where the forward rate is driven by a fractional Brownian motion.

Theorem 4.3. Suppose that $t \mapsto \sigma(t, T)$ is a continuous function not identically zero for all $T \in\left(0, T^{*}\right]$. Then the short rate process $(r(t))_{t \in\left[0, T^{*}\right]}$ is Markovian if and only if there exist continuous functions $\eta(t)$ and $\zeta(T)>0$ for $0<t<T \leq T^{*}$ such that $\sigma(t, T)$ admits the representation:

$$
\sigma(t, T)=-\frac{t^{1 / 2-H}}{2 H \Gamma(H+1 / 2) \Gamma(3 / 2-H)} \frac{d}{d t} \int_{t}^{T}(s-t)^{1 / 2-H} \eta(s) d s \cdot \zeta(T) .
$$

Proof. (i) Let us first assume that $(r(t))_{t \in\left[0, T^{*}\right]}$ is a Markov process. Then by virtue of (2.7) so is the process $(X(t))_{t \in\left[0, T^{*}\right]}$, and Lemma 4.1 shows that for each $0<T \leq S \leq T^{*}$ there is a measurable function $G$ such that (4.3) holds. 
Let us fix some $T \in\left(0, T^{*}\right]$. Then applying Lemma 4.2 to the functions $t \mapsto \gamma\left(t, T^{*}\right)$ and $t \mapsto \gamma(t, T)$, we get that there exists a function $\xi\left(T, T^{*}\right)$, not depending on $t$, such that:

$$
\gamma\left(t, T^{*}\right)=\xi\left(T, T^{*}\right) \cdot \gamma(t, T)
$$

for all $0<t<T \leq T^{*}$. Since for each $T \in\left(0, T^{*}\right]$ the function $t \mapsto \sigma(t, T)$ is not identically zero, so is the function $t \mapsto \gamma(t, T)$, and thus, there exists some $t \in(0, T)$ such that $\gamma(t, T) \neq 0$. Hence, the expression (4.6) implies that $\gamma\left(t, T^{*}\right) \neq 0$, so that $\xi\left(T, T^{*}\right) \neq 0$ for all $T \in\left(t, T^{*}\right]$. (Otherwise, if $\gamma\left(t, T^{*}\right)=0$, then $\xi\left(T, T^{*}\right)=0$, and from (4.6) it follows that the function $t \mapsto \gamma\left(t, T^{*}\right)$ is identically zero, which is a contradiction.) Since these arguments can be realized for each $T \in\left(0, T^{*}\right]$, we may conclude that $\xi\left(T, T^{*}\right) \neq 0$ for all $T \in\left(0, T^{*}\right]$.

Thus, setting $\eta(t)=\gamma\left(t, T^{*}\right)$ and $\zeta(T)=1 / \xi\left(T, T^{*}\right)$, we get the decomposition:

$$
\gamma(t, T)=\eta(t) \cdot \zeta(T)
$$

and hence, combining (3.9) with (4.7), we obtain that the expression:

$$
2 H(H-1 / 2) \int_{t}^{T} \sigma(s, T) s^{H-1 / 2}(s-t)^{H-3 / 2} d s=\eta(t) \cdot \zeta(T)
$$

holds for all $0<t<T \leq T^{*}$. We also observe that in this case without loss of generality we may assume that $\zeta(T)>0$ for all $T \in\left(0, T^{*}\right]$. The continuity of the functions $\eta(t), t \in(0, T)$, and $\zeta(T), T \in\left(0, T^{*}\right]$, directly follows from the continuity of $\gamma(t, T), 0<t<T \leq T^{*}$. Therefore, using the formula (3.13), from (4.8) by means of the same arguments as in Section 3 above we obtain that $\sigma(t, T)$ is given by $(4.5)$.

(ii) In order to prove the inverse assertion let us now assume that the function $\sigma(t, T)$ satisfies (4.8) with some $\eta(t)$ and $\zeta(T)>0$ and set:

$$
\beta(t)=\frac{t^{H-1 / 2}}{2 H(H-1 / 2) \Gamma^{2}(H-1 / 2) \Gamma^{2}(3 / 2-H)} \int_{0}^{t} s^{1-2 H}(t-s)^{H-3 / 2} \eta(s) d s .
$$

Then changing the order of integration, we get:

$$
\begin{aligned}
& \int_{0}^{t}(t-s)^{1 / 2-H} \int_{0}^{s} u^{1-2 H}(s-u)^{H-3 / 2} \eta(u) d u d s \\
& =\int_{0}^{t} u^{1-2 H} \eta(u) \int_{u}^{t}(t-s)^{1 / 2-H}(s-u)^{H-3 / 2} d s d u
\end{aligned}
$$

and thus, using the fact that the last inner integral in (4.10) equals $\Gamma(H-1 / 2) \Gamma(3 / 2-H)$, by means of straightforward calculations we obtain:

$$
\eta(t)=2 H \Gamma(H+1 / 2) \Gamma(3 / 2-H) t^{2 H-1} \frac{d}{d t} \int_{0}^{t} s^{1 / 2-H}(t-s)^{1 / 2-H} \beta(s) d s .
$$

Substituting the expression (4.11) for $\eta(t)$ into (4.5), we get that $\sigma(t, T)$ takes the form:

$$
\sigma(t, T)=\kappa(t, T ; \beta(\cdot)) \cdot \zeta(T)
$$


where we set:

$$
\kappa(t, T ; \beta(\cdot))=-t^{1 / 2-H} \frac{d}{d t} \int_{t}^{T} d s s^{2 H-1}(s-t)^{1 / 2-H} \frac{d}{d s} \int_{0}^{s} d u u^{1 / 2-H}(s-u)^{1 / 2-H} \beta(u) .
$$

It therefore follows that the process (4.1) admits the representation:

$$
X(t)=\zeta(t) \int_{0}^{t} \kappa(s, t ; \beta(\cdot)) d Z_{s}
$$

from where, by virtue of $[16$; Lemma 4$]$, we obtain that the process $(X(t) / \zeta(t))_{t \in\left[0, T^{*}\right]}$ is a Gaussian martingale (with independent increments). Hence, we may conclude that $(X(t))_{t \in\left[0, T^{*}\right]}$ is Markovian and so is the process $(r(t))_{t \in\left[0, T^{*}\right]}$.

Remark 4.4. Observe that if in (4.12) - (4.13) we have $\beta(t)=\beta=$ const for all $0<t<$ $T \leq T^{*}$, then by means of [16; Example 1] (see also [21]) we get that the volatility $\sigma(t, T$ ) takes the form:

$$
\sigma(t, T)=\beta \frac{\Gamma(3 / 2-H) \Gamma(2 H+1) \sin (\pi H)}{2 H \Gamma(H+1 / 2)} t^{1 / 2-H}(T-t)^{1 / 2-H} \cdot \zeta(T) .
$$

\section{Portfolios and arbitrage}

Following the arguments in [1], [2] and [26; Chapter VII, Section 5] we now introduce the notions of admissible self-financing portfolios for the bond market model introduced in Section 2. For this, let us introduce the discounted bond price process $\bar{P}=(\bar{P}(t, T))_{t \in[0, T]}$ defined by $\bar{P}(t, T)=P(t, T) / B(t)$ for all $0 \leq t \leq T \leq T^{*}$. We will consider only the so-called Markovian type portfolios, i.e. depending only on the current prices of bonds.

A pair $\pi=\left(g_{t}, h_{t}(d T)\right)_{t \in\left[0, T^{*}\right]}$ will be called a portfolio on the fractional bond market when for each Borel set $A \subseteq\left[t, T^{*}\right]$ fixed $g_{t}=g_{t}(\bar{P}(t, \cdot))$ and $h_{t}(A)=h_{t}(\bar{P}(t, \cdot), A)$ are $C^{1}$-functions of $\bar{P}(t, \cdot)$, and for each $\bar{P}(t, \cdot)$ fixed the function $h_{t}(d T)=h_{t}(\bar{P}(t, \cdot), d T)$ is a signed (finite) Borel measure on $\left[t, T^{*}\right]$. Here $g_{t}$ denotes the number of units held in the money account $B$, and $h_{t}(d T)$ denotes the number of bonds $P(t, \cdot)$ with maturities in the interval $[T, T+d T]$ held in the portfolio $\pi$ at time $t$.

A portfolio $\pi=\left(g_{t}, h_{t}(d T)\right)_{t \in\left[0, T^{*}\right]}$ is said to be feasible if the conditions:

$$
\int_{0}^{T^{*}}\left(\left|g_{t}\right|+\int_{t}^{T^{*}}|\alpha(t, T)|\left|h_{t}(d T)\right|\right) d t<\infty
$$

and

$$
\int_{0}^{T^{*}} \int_{0}^{T^{*}} \int_{t}^{T^{*}}|\sigma(t, T)|\left|h_{t}(d T)\right| \int_{s}^{T^{*}}|\sigma(s, S)|\left|h_{s}(d S)\right||t-s|^{2 H-2} d s d t<\infty
$$

hold (for the integration of measure-valued processes cf. [1] and [2]). We will say that a feasible portfolio $\pi=\left(g_{t}, h_{t}(d T)\right)_{t \in\left[0, T^{*}\right]}$ is admissible if its value process $V=\left(V_{t}\right)_{t \in\left[0, T^{*}\right]}$ defined by:

$$
V_{t}=g_{t} B(t)+\int_{t}^{T^{*}} P(t, T) h_{t}(d T)
$$


satisfies the condition:

$$
V_{t} / B(t) \geq-a
$$

for some $a \geq 0$ and all $0 \leq t \leq T^{*}$. A feasible portfolio $\pi=\left(g_{t}, h_{t}(d T)\right)_{t \in\left[0, T^{*}\right]}$ is said to be self-financing if the value process $V$ admits the representation:

$$
V_{t}=V_{0}+\int_{0}^{t} g_{s} d B(s)+\int_{0}^{t} \int_{s}^{T^{*}} d P(s, T) h_{s}(d T)
$$

where the integral with respect to $d P(s, T)$ is well-defined in the pathwise sense (see e.g. [9], [10], [17] and [31]). We will say that an (admissible self-financing) portfolio realizes arbitrage on the time interval $\left[0, T^{*}\right]$ if for its value process $V$ the following conditions are satisfied:

$$
V_{0}=0, \quad P\left[V_{T^{*}} \geq 0\right]=1 \quad \text { and } \quad P\left[V_{T^{*}}>0\right]>0 .
$$

On the given fractional bond market we now construct an arbitrage portfolio having the same structure as the related arbitrage strategy on the fractional stock market in [26; Chapter VII, Section 2c]. For this, let us take a portfolio $\pi^{\prime}=\left(g_{t}^{\prime}, h_{t}^{\prime}(d T)\right)_{t \in\left[0, T^{*}\right]}$ such that:

$$
g_{t}^{\prime}=-\left[\bar{P}\left(t, T^{*}\right)-\bar{P}\left(0, T^{*}\right)\right]\left[\bar{P}\left(t, T^{*}\right)+\bar{P}\left(0, T^{*}\right)\right]
$$

and

$$
h_{t}^{\prime}(d T)=2[\bar{P}(t, T)-\bar{P}(0, T)] \delta_{\left\{T^{*}\right\}}(d T)
$$

where $\delta_{\left\{T^{*}\right\}}(d T)$ denotes the Dirac measure in the point $T^{*}$. Hence, the corresponding value process $V^{\prime}=\left(V_{t}^{\prime}\right)_{t \in\left[0, T^{*}\right]}$ started at $V_{0}^{\prime}=0$ is given by:

$$
V_{t}^{\prime}=V_{0}^{\prime}+g_{t}^{\prime} B(t)+\int_{t}^{T^{*}} P(t, T) h_{t}^{\prime}(d T)=\frac{1}{B(t)}\left[P\left(t, T^{*}\right)-P\left(0, T^{*}\right) B(t)\right]^{2}
$$

and by means of the classical change-of-variable formula, using the fact that the sample paths of the process $(P(t, T))_{t \in[0, T]}$ have zero quadratic variation, we obtain that the process $V^{\prime}$ admits the representation:

$$
\begin{aligned}
d V_{t}^{\prime}= & -\frac{1}{B^{2}(t)}\left[P\left(t, T^{*}\right)-P\left(0, T^{*}\right) B(t)\right]^{2} d B(t) \\
& +\frac{2}{B(t)}\left[P\left(t, T^{*}\right)-P\left(0, T^{*}\right) B(t)\right]\left[d P\left(t, T^{*}\right)-P\left(0, T^{*}\right) d B(t)\right] .
\end{aligned}
$$

Therefore, from (5.10) it is easily seen that the portfolio $\pi^{\prime}=\left(g_{t}^{\prime}, h_{t}^{\prime}(d T)\right)_{t \in\left[0, T^{*}\right]}$ is self-financing, and from (5.9) we conclude that it is admissible and realizes arbitrage in the sense of (5.6).

Remark 5.1. We observe that inserting the expression (4.5) for $\sigma(t, T)$ into (2.7), by means of the arguments in the part (ii) of the proof of Theorem 4.3 we may conclude that the short rate process $(r(t))_{t \in\left[0, T^{*}\right]}$ is a semimartingale. The same time, the forward rate process $(f(t, T))_{t \in[0, T]}$ and the bond price process $(P(t, T))_{t \in[0, T]}$ are not semimartingales, and the arguments above show that the bond market model admits an arbitrage opportunity.

Acknowledgments. This paper was partially written and finished during the author's visits to the University of Helsinki in November 1999, August 2000 and September 2003, and he is grateful to Esko Valkeila for the hospitality and many useful comments. The author is pleased to thank Freddy Delbaen for many useful discussions concerning fractional markets and Uwe Küchler for many fruitful discussions concerning term structure models. 


\section{References}

[1] Buörk, T., Kabanov, Yu. M., and Runggaldier, W. (1997). Bond market structure in the presence of marked point processes. Mathematical Finance 7(2) (211-239).

[2] Björk, T., Di Masi, G., Kabanov, Yu. M., and Runggaldier, W. (1997). Towards a general theory of bond markets. Finance and Stochastics 1(2) (141-174).

[3] Carverhill, A. (1994). When is the short rate Markovian? Mathematical Finance 4(4) $(305-312)$.

[4] Cheridito, P. (2003). Arbitrage in fractional Brownian motion models. Finance and Stochastics 7(4) (533-553).

[5] Cheridito, P. (2003). Gaussian moving averages, semimartingales and option pricing. To appear in Stochastic Processes and Applications (22 pp).

[6] Decreusefond, L. and Üstünel, A. S. (1999). Stochastic analysis of the fractional Brownian motion. Potential Analysis 10 (177-214).

[7] Dasgupta, A. and Kallianpur, G. (2000). Arbitrage opportunities for a class of Gladyshev processes. Appl. Math. Opt. 41 (377-385).

[8] Eberlein, E. and Raible, S. (1999). Term structure models driven by general Lévy processes. Mathematical Finance 9(1) (31-53).

[9] Föllmer, H. (1981). Calcul d'Itô sans probabilités. Lecture Notes in Mathematics, Springer 850 (143-150).

[10] Föllmer, H., Protter, Ph. and Shiryaev, A. N. (1995). Quadratic covariation and an extension of Itô's formula. Bernoulli 1 (149-169).

[11] Gapeev, P. V. and Küchler, U. (2003). On Markovian short rates in term structure models driven by jump-diffusion processes. Discussion Paper 44(2003) of Sonderforschungsbereich 373, Humboldt University Berlin (16 pp).

[12] Gripenberg, G. and Norros, I. (1996). On the prediction of fractional Brownian motion. J. Appl. Probab. 33 (400-410).

[13] Heath, D., Jarrow, R. and Morton, A. (1992). Bond pricing and the term structure of interest rates: A new methodology for contingent claims valuation. Econometrica 60(1) (77-105).

[14] Kolmogorov, A. N. (1941). Wienersche Spiralen und einige andere interessante Kurven im Hilbertschen Raum. Comptes Rendus (Doklady) Acad. Sci. USSR 26 (115-118).

[15] KüChler, U. and Naumann, E. (2003). Markovian short rates in a forward rate model with a general class of Lévy processes. Discussion Paper 6(2003) of Sonderforschungsbereich 373, Humboldt University Berlin (20 pp). 
[16] Le Breton, A. (1998). Filtering and parameter estimation in a simple linear system driven by a fractional Brownian motion. Statistics and Probability Letters 38 (263-274).

[17] Lin, J. S. (1995). Stochastic analysis of fractional Brownian motion. Stochastics and Stochastic Reports 55 (121-140).

[18] Liptser, R. S. and Shiryaev, A. N. (1989). Theory of Martingales. Kluwer, Dordrecht.

[19] Mandelbrot, B. and van Ness, J. (1968). Fractional Brownian motions, fractional noises and applications. SIAM Review 10 (422-437).

[20] Molchan, G. (1969). Gaussian processes with spectra which are asymptotically equivalent to a power of $\lambda$. Theory of Probab. Appl. 14 (530-532).

[21] Norros, I., Valkeila, E. and Virtamo, J. (1999). An elementary approach to a Girsanov formula and other analytical results on fractional Brownian motion. Bernoulli 5(4) (571-587).

[22] NorvaišA, R. (2000). Modelling of stock price changes: a real analysis approach. Finance and Stochastics 4 (343-369).

[23] Rogers, L. C. G. (1997). Arbitrage with fractional Brownian motion. Mathematical Finance 7(1) (95-105).

[24] SalopeK, D. M. (1998). Tolerance to arbitrage. Stochastic Processes and their Applications 76 (217-230).

[25] Samorodnitsky, G. and Taqqu, M. S. (1994). Stable Non-Gaussian Random Processes: Stochastic Models with Infinite Variance. Chapman and Hall, London.

[26] Shiryaev, A. N. (1999). Essentials of Stochastic Finance. World Scientific, Singapore.

[27] Sottinen, T. (2001). Fractional Brownian motion, random walks, and binary market models. Finance and Stochastics 5(3) (343-355).

[28] Sottinen, T. and Valkeila, E. (2001). Fractional Brownian motion as a model in finance. University of Helsinki, Department of Mathematics, Preprint 302 (16 pp).

[29] Sottinen, T. and Valkeila, E. (2003). On arbitrage and replication in the fractional Black-Scholes pricing model. Statistics and Decisions 21 (93-107).

[30] Valkeila, E. (1999). On some properties of geometric fractional Brownian motions. University of Helsinki, Department of Mathematics, Preprint 224 (12 pp).

[31] Z̈̈HLE, M. (1997). Integration with respect to fractal functions and stochastic calculus. Probability Theory and Related Fields 111 (333-374). 